\title{
SYMMETRY REDUCTION OF UNSTEADY MHD ALIGNED SECOND GRADE FLOW EQUATIONS
}

\author{
Asif Ali and Ahmer Mehmood \\ Department of Mathematics, Quaid-I-Azam University 45320 \\ Islamabad - 44000, Pakistan
}

\begin{abstract}
Exact solutions for the unsteady flow equations of an incompressible MHD aligned second grade fluid are obtained. Translational symmetries are used successively to reduce the governing partial differential equations into ordinary differential equations.
\end{abstract}

Keywords- Unsteady plane flow, MHD aligned fluid, translational symmetry, exact solutions.

\section{INTRODUCTION}

The differential equations governing the flow of non-Newtonian second grade fluids are highly nonlinear in nature. The nonlinearities occur in inertial as well as in viscosity parts [1, 2]. For a homogeneous incompressible second grade fluid, the governing equations are one order higher than the Navier-Stokes equations, that's why an additional boundary condition is required. However, there are many flow situations for which there are no such restrictions. For details reader is referred to study [3-7].

Inverse methods [8-14] play an important role to solve the general flow equations exactly, but unfortunately these solutions are not the general solution of the original equations because these are obtained by assuming a special form of the stream function. The form of the stream function is assumed due to the flow geometry of the flow field.

Due to the non-availability of the techniques which could be applied to the nonlinear equations directly, we seek some transformations which lead to the simplification of the nonlinear equations. These transformations leave the equations invariant. Sophus Lie gave a systematic approach to find the symmetries of differential equations which was further improved by several authors. For details the reader is referred to see the references [15-19]. Symmetries can reduce the order as well as the number of independent variables appearing in the differential equations [20-22].

In the present analysis we have considered the unsteady two directional, two dimensional flow equations for an incompressible MHD aligned second grade fluid. The same equations were solved by inverse method exactly by Chandna [11]; the solutions obtained by Chandna are however exact but not general whereas the solutions obtained by us are the general solutions. Translational symmetries are used twice to reduce the partial differential equations into ordinary differential equations. 


\section{FLOW EQUATIONS}

The basic equations for an incompressible MHD aligned second grade fluid are

$$
\begin{gathered}
\nabla \cdot \mathbf{V}=0, \quad \text { (continuity) } \\
\rho\left[\mathbf{V}_{t}+(\mathbf{V} \cdot \nabla) \mathbf{V}\right]=\nabla \cdot \mathbf{T}+\rho \mathbf{b}+\mu^{*}(\nabla \times \mathbf{H}) \times \mathbf{H},(\text { linear momentum) } \\
\mathbf{H}_{t}=\nabla \times(\nabla \times \mathbf{H})-\frac{1}{\mu^{*} \sigma} \nabla \times(\nabla \times \mathbf{H}), \quad(\text { diffusion) } \\
\nabla \cdot \mathbf{H}=0, \quad \text { (solenoidal) }
\end{gathered}
$$

The constitutive equation for the stress of a second grade fluid [7] is

$$
\mathbf{T}=-p \mathbf{I}+\mu \mathbf{A}_{1}+\alpha_{1} \mathbf{A}_{2}+\alpha_{2} \mathbf{A}_{1}^{2},
$$

with

$$
\begin{gathered}
\mathbf{A}_{1}=\mathbf{L}+\mathbf{L}^{t}, \quad \mathbf{L}=\nabla \mathbf{V}, \\
\mathbf{A}_{2}=\left(\frac{\partial}{\partial t}+\mathbf{V} \cdot \nabla\right) \mathbf{A}_{1}+\mathbf{L} \mathbf{V}+\mathbf{L}^{T} \mathbf{A}_{1},
\end{gathered}
$$

where $\mathbf{V}$ is the velocity vector, $\mathrm{b}$ is the body force per unit mass, $\mathbf{H}$ is the magnetic field intensity, $\mathrm{p}$ is the pressure, $\rho$ is the fluid density, $v$ is the magnetic permeability, $\sigma$ is the electrical conductivity, $\mu$ is the constant viscosity, $\alpha_{1}$ and $\alpha_{2}$ are the constant normal stress moduli, $\mathbf{A}_{1}$ and $\mathbf{A}_{2}$ are the first and second Rivlin-Ericksen tensors respectively. The solenoidal equation (4) shows that there is no magnetic pole in the flow field. follows;

On specializing velocity, magnetic field intensity and pressure distribution are as

$$
\mathbf{V}=[u(x, y, t), v(x, y, t), 0], \quad \mathbf{H}=\left[H_{1}(x, y, t), H_{2}(x, y, t), 0\right] \quad \text { and } \quad p=p(x, y, t)
$$

We get the following equations from Eqs. (1) - (4) after neglecting the body forces

$$
\begin{gathered}
u_{x}+v_{y}=0, \\
u_{t}+u_{x}+v u_{y}+\frac{1}{\rho} p_{x}=v\left(u_{x x}+u_{y y}\right)+\alpha\left[\begin{array}{l}
u_{x x t}+u_{y y t}+u u_{x x x}+13 u_{x} u_{x x}+v u_{x x y} \\
+4 v_{x} v_{x x}+2 v_{x} u_{x y}+3 v_{x x} u_{y}+u u_{x y y} \\
+3 u_{y} u_{x y}+v u_{y y y}+u_{x} u_{y y}
\end{array}\right] \\
+\beta\left[8 u_{x} u_{x x}+2 v v_{x x}+2 u_{y} u_{x y}+2 v_{x x} u_{y}+2 v_{x} u_{x y}\right]-v^{*} H_{2}\left(H_{2 x}-H_{1 y}\right), \\
v_{t}+u v_{x}-v u_{x}+\frac{1}{\rho} p_{y}=v\left(v_{x x}-u_{x y}\right)+\alpha\left[\begin{array}{l}
v_{x x t}-u_{x y t}+u v_{x x x}-u_{x} v_{x x}-u u_{x x y} \\
+13 u_{x} u_{x y}-v u_{x x x}-3 u_{x x} v_{x}+3 v_{x} u_{y y} \\
-v u_{x y y}-2 u_{x x} u_{y}+4 u_{y} u_{y y}
\end{array}\right] \\
+\beta\left[8 u_{x} u_{x y}-2 v_{x} u_{x x}+2 u_{y} u_{y y}+2 v_{x} u_{y y}-2 u_{x x} u_{y}\right]+v^{*} H_{1}\left(H_{2 x}-H_{1 y}\right), \\
H_{2 x t}-H_{1 y t}=\frac{1}{\mu^{*} \sigma}\left[H_{2 x x x}+H_{2 x y y}-H_{1 x x y}-H_{1 y y y}\right]+v H_{1 x x}+v_{x x} H_{1}+v H_{1 y y} \\
+v_{y y} H_{1}-u H_{2 x x}-u_{x x} H_{2}-u H_{2 y y}-u_{y y} H_{2}
\end{gathered}
$$




$$
H_{1 x}+H_{2 y}=0
$$

where $v=\mu / \rho, \quad v^{*}=\mu^{*} / \rho, \quad \alpha=\alpha_{1} / \rho \quad$ and $\beta=\alpha_{2} / \rho$.

\section{SOLUTION OF THE PROBLEM}

Equations (7)-(11) are a system of nonlinear partial differential equations with five dependent and three independent variables. It is not easy to get the exact solution of this system in this form. Rivlin and Ericksen [1] used the inverse method to get the solutions of the same problems by assuming a special form of the stream function, these solutions are however exact but not general. We use the one-parameter Lie point symmetries to reduce the system of partial differential equations into a system of ordinary differential equations. For this purpose we use the translational symmetries twice. The system (7) - (11) admits the translational symmetries in $\mathrm{x}-$, y- and $\mathrm{t}-$ directions. First we use the combination of $\partial / \partial x$ and $\partial / \partial y$ which lead the following transformations for the dependent and independent variables

$$
\xi=y-a x, u=f^{*}(\xi, t), v=g^{*}(\xi, t), p=h^{*}(\xi, t), H_{1}=L_{1}^{*}(\xi, t) \text { and } H_{2}=L_{2}{ }^{*}(\xi, t) \text {, }
$$

where $a$ is an arbitrary constant.

Therefore the system (7)-(11) reduces to the following system of partial differential equations

$$
\begin{gathered}
g^{*}{ }_{\xi}-a f^{*}{ }_{\xi}=0, \\
f^{*}+\left(g^{*}-a f^{*}\right) f^{*}{ }_{\xi}-\frac{a}{\rho} h^{*} \xi=v\left(1+a^{2}\right) f^{*}{ }_{\xi \xi}+a\left(1+a^{2}\right)\left[\begin{array}{l}
f^{*}{ }_{\xi \xi t}-a f^{*} f^{*}{ }_{\xi \xi \xi} \\
+g^{*} f^{*}{ }_{\xi \xi \xi}
\end{array}\right] \\
-2 a(2 \alpha+\beta)\left(1+a^{2}\right)^{2} f^{*}{ }_{\xi} f^{*}{ }_{\xi \xi}+v^{*} L_{2}{ }^{*}\left(a L_{2 \xi}{ }^{*}+L_{1 \xi}{ }^{*}\right), \\
g_{t}{ }^{*}+a\left(g^{*}-a f^{*}\right) f^{*}{ }_{\xi}+\frac{a}{\rho} h^{*}{ }_{\xi}=v a\left(1+a^{2}\right) f^{*}{ }_{\xi \xi}+a\left(1+a^{2}\right)\left[\begin{array}{l}
a f^{*}{ }_{\xi \xi t}-a^{2} f^{*} f^{*}{ }_{\xi \xi \xi} \\
+a g^{*} f^{*}{ }_{\xi \xi \xi}
\end{array}\right] \\
+2(2 \alpha+\beta)\left(1+a^{2}\right)^{2} f^{*}{ }_{\xi} f^{*}{ }_{\xi \xi}-v^{*} L_{1}^{*}\left(a L^{*}{ }_{2 \xi}+L^{*}{ }_{1 \xi}\right), \\
L^{*}{ }_{1 \xi t}=\frac{\left(1+a^{2}\right)}{\mu^{*} \sigma} L^{*}{ }_{1 \xi \xi \xi}+\left(g^{*}-a f^{*}\right) L_{1 \xi \xi}^{*}+\left(a L_{1}^{*}-L^{*}{ }_{2}\right) f^{*}{ }_{\xi \xi \xi}, \\
L^{*}{ }_{2 \xi}-a L^{*}{ }_{1 \xi}=0,
\end{gathered}
$$

The system (13) - (17) admits the symmetries $\partial / \partial \xi$ and $\partial / \partial t$. We use the combination of these two symmetries to reduce the above system of partial differential equations into a system of ordinary differential equations. Therefore, $\partial / \partial \xi+b \partial / \partial t$ leads to the following change of variables

$\eta=\xi-b t, f^{*}=f(\xi), \quad g^{*}=g(\eta), \quad h^{*}=h(\eta), \quad L_{1}^{*}=L_{1}(\eta), L_{2}^{*}=L_{2}(\eta)$

where $b$ is an arbitrary constant.

Using the transformations given in Eq. (18), the system (13) - (17) reduces to

$$
g^{\prime}-b f^{\prime}=0 \text {, }
$$




$$
\begin{aligned}
& -b f^{\prime}+(g-a f) f^{\prime}-\frac{a}{\rho} h^{\prime}=v\left(1+a^{2}\right) f^{\prime \prime}+a\left(1+a^{2}\right)\left[-b f^{\prime \prime \prime}-a f f^{\prime \prime \prime}+g f^{\prime \prime \prime}\right] \\
& -2 a(2 \alpha+\beta)\left(1+a^{2}\right)^{2} f^{\prime} f^{\prime \prime}+v^{*}\left(1+a^{2}\right) L_{1}^{\prime} L_{2}, \\
& -b g^{\prime}+a(g-a f) f^{\prime}+\frac{1}{\rho} h^{\prime}=v a\left(1+a^{2}\right) f^{\prime \prime}+\alpha\left(1+a^{2}\right)\left[-a b f^{\prime \prime \prime}-a^{2} f f^{\prime \prime \prime}+a g f^{\prime \prime \prime}\right] \\
& +2(2 \alpha+\beta)\left(1+a^{2}\right)^{2} f^{\prime} f^{\prime \prime}-v^{*}\left(1+a^{2}\right) L_{1} L_{1}^{\prime}, \\
& -b L_{1}^{\prime \prime}=\frac{1+a^{2}}{\mu^{*} \sigma} L_{1}^{\prime \prime \prime}-(g-a f) L_{1}^{\prime \prime}+\left(a L_{1}-L_{2}\right) f^{\prime \prime}, \\
& L_{2}^{\prime}-a L_{1}^{\prime}=0,
\end{aligned}
$$

Hence the system (7) - (11) of partial differential equations is reduced to the system (19) - (23) of ordinary differential equations. From Eqs. (19) - (23) we have

$$
\begin{gathered}
g=a f+c_{1}, \\
L_{2}=a L_{1}+c_{2},
\end{gathered}
$$

Multiplying Eq. (21) by $a$ and then adding it to Eq. (20) and using Eq. (24) and (25) we get

$$
a\left(1+a^{2}\right)\left(c_{1}-b\right) f^{\prime \prime \prime}+v\left(1+a^{2}\right) f^{\prime \prime}-\left(c_{1}-b\right) f^{\prime}=0,
$$

Here two cases arise for Eq. (26)

I $c_{1}=b$

II $c_{1} \neq b$

3.1 Case I $\left(c_{1}=b\right)$

In this case Eq. (26) takes the form

$$
f^{\prime \prime}=0
$$

which on integration gives

Using Eq. (27) in Eq. (24) we find

$$
f=c_{2} \eta+c_{4},
$$

Substituting Eqs. (24), (25) and (27) into Eq. (22) we have

which on integration gives

$$
L_{1}^{\prime \prime \prime}=0 \text {, }
$$

$$
L_{1}=c_{5} \eta^{2}+c_{6} \eta+c_{7}
$$

Using Eq. (29) in Eq. (25) we get

$$
L_{2}=a\left(c_{5} \eta^{2}+c_{6} \eta+c_{7}\right)+c_{2} .
$$

Eq. (21) with Eq. (24) becomes

$$
h^{\prime}=\left(1+a^{2}\right)\left[a \mu f^{\prime \prime}+2\left(2 \alpha_{1}+\alpha_{2}\right)\left(1+a^{2}\right) f^{\prime} f^{\prime \prime}-v^{*} L_{1} L_{1}^{\prime}\right]+\rho b c_{1},
$$

which on integration gives

$$
h=\left(1+a^{2}\right)\left[\mu a c_{3} \eta+2\left(\alpha_{1}+\alpha_{2}\right)\left(1+a^{2}\right) c_{3}^{2} \eta^{2}-\frac{\mu}{2}\left(c_{5} \eta^{2}+c_{6} \eta+c_{7}\right)\right]+\rho b c_{1} \eta+c_{8},
$$

where $\mathrm{C}_{\mathrm{i}}$ 's $i=1 \ldots 8$ are the constants of integration. 
Making use of back substitution consecutively with Eqs. (18) and (12), Eqs. (27) - (31) can be written in the form of original variables

$$
\begin{gathered}
u(x, y, t)=c_{3}\left(y-a x-c_{1} t\right)+c_{4}, \\
v(x, y, t)=a c_{3}\left(y-a x-c_{1} t\right)+a c_{4}+c_{1}, \\
L_{1}(x, y, t)=c_{5}\left(y-a x-c_{1} t\right)^{2}+c_{6}\left(y-a x-c_{1} t\right)+c_{7}, \\
p(x, y, t)=\left(1+a^{2}\right)\left[\begin{array}{l}
\mu a c_{3}\left(y-a x-c_{1} t\right)+\rho b c_{1}\left(y-a x-c_{1} t\right) \\
-\frac{\mu}{2}\left(c_{5}\left(y-a x-c_{1} t\right)+c_{6}\left(y-a x-c_{1} t\right)\right)^{2} \\
+2\left(2 \alpha_{1}+\alpha_{2}\right)\left(1+a^{2}\right) c_{3}^{2}\left(y-a x-c_{1} t\right)^{2}+c_{8}
\end{array}\right] .
\end{gathered}
$$

3.2 Case II $\left(c_{1} \neq b\right)$

For this case we have the following equation

$$
\alpha\left(1+a^{2}\right)\left(c_{1}-b\right) f^{\prime \prime \prime}+v\left(1+a^{2}\right) f^{\prime \prime}-\left(c_{1}-b\right) f^{\prime}=0,
$$

which admits the solution

$$
f=\frac{c_{9}}{d_{1}} e^{d_{1} \eta}+\frac{c_{10}}{d_{2}} e^{d_{2} \eta}+c_{11}
$$

where

$$
d_{1,2}=-\frac{v}{2 \alpha\left(c_{1}-b\right)} \pm \frac{1}{2} \sqrt{\frac{v^{2}}{\alpha^{2}\left(c_{1}-b\right)^{2}}+\frac{4}{\alpha\left(1+a^{2}\right)}} .
$$

Using Eq. (37) in Eq. (24) we have

$$
g=a\left(\frac{c_{9}}{d_{1}} e^{d_{1} \eta}+\frac{c_{10}}{d_{2}} e^{d_{2} \eta}+c_{11}\right)+c_{1} .
$$

Using Eqs. (24), (25) and (37) in Eq. (22) we get

$$
L_{1}^{\prime \prime \prime}+\lambda L_{1}^{\prime \prime}=\frac{\lambda}{b-c_{1}} c_{2} f^{\prime \prime},
$$

which has the following solution

$$
L_{1}=\frac{\lambda}{b-c_{1}} c_{2}\left[\frac{c_{9}}{d_{1}\left(d_{1}-\lambda\right)} e^{d_{1} \eta}+\frac{c_{10}}{d_{2}\left(d_{2}+\lambda\right)} e^{d_{2} \eta}\right]+\frac{c_{12}}{\lambda^{2}} e^{-\lambda \eta}+c_{13} \eta+c_{14},
$$

where $\lambda=\mu * \sigma\left(b-c_{1}\right) /\left(1+a^{2}\right)$

Substituting Eq. (41) into Eq. (25) we get

$$
L_{2}=\frac{a \lambda}{b-c_{1}} c_{2}\left[\frac{c_{9}}{d_{1}\left(d_{1}+\lambda\right)} e^{d_{1} \eta}+\frac{c_{10}}{d_{2}\left(d_{2}+\lambda\right)} e^{d_{2} \eta}\right]+\frac{a c_{12}}{\lambda^{2}} e^{-\lambda \eta}+a c_{13} \eta+a c_{14} .
$$

From Eq. (15) we have

$$
\begin{aligned}
& h^{\prime}=\rho a\left(b-c_{1}\right) f+\mu a\left(1+a^{2}\right) f^{\prime \prime}+a \alpha_{1}\left(1+a^{2}\right)\left(b-c_{1}\right) f^{\prime \prime \prime}+\left(2 \alpha_{1}+\alpha_{2}\right)\left(1+a^{2}\right)^{2} f^{\prime} f^{\prime \prime} \\
& -\mu *\left(1+a^{2}\right) L_{1} L_{1}{ }^{\prime},
\end{aligned}
$$

Integration of the above equation yields 


$$
\begin{aligned}
& h=\left[\frac{\rho a\left(b-c_{1}\right)}{d_{1}}+\left(1+a^{2}\right)\left(\mu a+a \alpha_{1}\left(b-c_{1}\right) d_{1}\right)\right] c_{9} e^{d_{1} \eta} \\
& +\left[\frac{\rho a\left(b-c_{1}\right)}{d_{2}}+\left(1+a^{2}\right)\left(\mu a+a \alpha_{1}\left(b-c_{1}\right) d_{2}\right)\right] c_{10} e^{d_{2} \eta} \\
& +\left(2 \alpha_{1}+\alpha_{2}\right)\left(1+a^{2}\right)^{2}\left[c_{9}^{2} e^{2 d_{1} \eta}+c_{10}^{2} e^{2 d_{2} \eta}+2 c_{9} c_{10} e^{\left(d_{1}+d_{2}\right) \eta}\right] \\
& -\frac{\mu^{*}\left(1+a^{2}\right)}{2}\left[\frac{\lambda}{b-c_{1}}\left(\frac{c_{9}}{d_{1}\left(d_{1}+\lambda\right)} e^{d_{1} \eta}+\frac{c_{10}}{d_{2}\left(d_{2}+\lambda\right)} e^{d_{2} \eta}\right)\right]^{2}, \\
& +\frac{c_{12}}{\lambda^{2}} e^{-\lambda \eta}+c_{13} \eta+c_{14}
\end{aligned}
$$

where $\mathrm{C}_{\mathrm{i}}$ 's $i=8 \ldots 14$ are the constants of integration.

Making use of back substitution in Eqs. (37), (39), (41), (42) and (44) from Eqs. (18) and (12) respectively, we get the solution in the form of original variables

$$
\begin{gathered}
u(x, y, t)=\frac{c_{9}}{d_{1}} e^{d_{1}\left(y-a x-c_{1} t\right)}+\frac{c_{10}}{d_{2}} e^{d_{2}\left(y-a x-c_{1} t\right)}+c_{11}, \\
v(x, y, t)=a\left(\frac{c_{9}}{d_{1}} e^{d_{1}\left(y-a x-c_{1} t\right)}+\frac{c_{10}}{d_{2}} e^{d_{2}\left(y-a x-c_{1} t\right)}+c_{11}\right)+c_{1}, \\
H_{1}(x, y, t)=\frac{\lambda}{b-c_{1}} c_{2}\left[\frac{c_{9}}{d_{1}\left(d_{1}+\lambda\right)} e^{d_{1}\left(y-a x-c_{1} t\right)}+\frac{c_{10}}{d_{2}\left(d_{2}+\lambda\right)} e^{d_{2}\left(y-a x-c_{1} t\right)}\right] \\
+\frac{c_{12}}{\lambda^{2}} e^{-\lambda\left(y-a x-c_{1} t\right)}+c_{13}\left(y-a x-c_{1} t\right)+c_{14}, \\
H_{2}(x, y, t)=\frac{a \lambda}{b-c_{1}} c_{2}\left[\frac{c_{9}}{d_{1}\left(d_{1}+\lambda\right)} e^{d_{1}\left(y-a x-c_{1} t\right)}+\frac{c_{10}}{d_{2}\left(d_{2}+\lambda\right)} e^{d_{2}\left(y-a x-c_{1} t\right)}\right] \\
+\frac{a c_{12}}{\lambda^{2}} e^{-\lambda\left(y-a x-c_{1} t\right)}+a c_{13}\left(y-a x-c_{1} t\right)+a c_{14},
\end{gathered}
$$




$$
\begin{aligned}
& p(x, y, t)=\left[\frac{\rho a\left(b-c_{1}\right)}{d_{1}}+\left(1+a^{2}\right)\left(\mu a+\alpha_{1} a\left(b-c_{1}\right) d_{1}\right)\right] c_{9} e^{d_{1}\left(y-a x-c_{1} t\right)} \\
& +\left[\frac{\rho a\left(b-c_{1}\right)}{d_{2}}+\left(1+a^{2}\right)\left(\mu a+\alpha_{1} a\left(b-c_{1}\right) d_{2}\right)\right] c_{10} e^{d_{2}\left(y-a x-c_{1} t\right)} \\
& +\left(2 \alpha_{1}+\alpha_{2}\right)\left(1+a^{2}\right)\left[c_{9}^{2} e^{2 d_{1}\left(y-a x-c_{1} t\right)}+c_{10}{ }^{2} e^{2 d_{2}\left(y-a x-c_{1} t\right)}+2 c_{9} c_{10} e^{\left(d_{1}+d_{2}\right)\left(y-a x-c_{1} t\right)}\right] \\
& -\frac{\mu^{*}\left(1+a^{2}\right)}{2}\left[\begin{array}{c}
\frac{\lambda}{b-c_{1}}\left(\begin{array}{c}
\frac{c_{9}}{d_{1}\left(d_{1}+\lambda\right)} e^{d_{1}\left(y-a x-c_{1} t\right)} \\
+\frac{c_{10}}{d_{2}\left(d_{2}+\lambda\right)} e^{d_{2}\left(y-a x-c_{1} t\right)}
\end{array}\right) \\
+\frac{c_{12}}{\lambda^{2}} e^{-\lambda\left(y-a x-c_{1} t\right)}+c_{13}\left(y-a x-c_{1} t\right)+c_{14}
\end{array}\right]^{2}
\end{aligned}
$$

\section{CONCLUDING REMARKS}

Exact solutions to the nonlinear differential equations governing the flow of an incompressible MHD aligned second grade fluid were obtained by symmetry method. The symmetries of translational type were used. It was observed that the translational symmetries had led to exponential type exact solutions, but in the case $\mathrm{I}\left(c_{1}=b\right)$, when both the velocity components $u$ and $v$ were proportional to each other the solutions were not of exponential type.

\section{REFERENCES}

1. R.S. Rivlin and J.L. Ericksen, stress deformation relation for isotropic materials. J. Rat. Mech. Anal. 4, 323 - 425, 1955.

2. B.D. Coleman, W. Noll, An approximation theorem for functionals with applications in continumm mechanics, Arc. Rat. Mech. Anal. 6, 335 - 370, 1955.

3. G.T. Taylor, on the decay of vorticies in viscous fluid, Phil. Mag. 46, $671-674$, 1923.

4. L.I.G. Kovasnay, Laminar flow behind a two dimensional grid, Proc. Cambridge Phil. Soc. 44, 58 - 62, 1948.

5. C.Y. Wang, On a class of exact solutions of the Navier Stokes equations, J. Appl. Mech. 33, 696 - 698, 1966.

6. S.P. Lin, M. Tobak, Reversed flow above a plate with suction, AIAA J. 24, 334 $335,1986$.

7. W.H. Hui, Exact solutions of the unsteady two dimensional Navier Stokes equations, ZAMP, 38, 689 - 702, 1987.

8. P.F. Nemeny, Recent developments in inverse and semi inverse methods in the mechanics of continua, Advances in Applied Mathematics, 2, New York, 1951.

9. P.N. Kaloni and K. Huschilt, Semi-inverse solutions of non-Newtonian fluid. Int. J. Non-Linear Mech. 19, 373 - 381, 1984. 
10. A.M. Siddiqui and P.N. Kaloni, Certain inverse solutions of non-Newtonian fluid, Int. J. non-Linear Mech. 21, 459 - 473, 1986.

11. O.P. Chandna and E.O. Oku-Ukpong, Unsteady second-grade aligned MHD fluid flow, Acta Mech. 107, 77 - 91, 1994.

12. M.R. Mohyuddin, T. Hayat, F.M. Mahomed, S. Asghar, and A.M. Siddiqui, On solutions of some non-linear differential equations arising in Newtonian and non-Newtonian fluids, Nonlinear Dynamics 35, 229 - 245, 2004.

13. A.M. Siddiqui, M.R. Mohyuddin, T. Hayat and S. Asghar, Some more inverse solutions for steady flows of a second-grade fluid, Arch. Mech. 55, 371, 2003.

14. F. Labropulu, Exact solutions of non-Newtonian fluid flow with prescribed vorticity, Acta Mech. 141, $11-20,2000$.

15. G.W. Bluman and S. Kumei, Symmetries and differential equations, Springer, New York, 1998.

16. H. Stephini, Differential equations, their solutions using symmetries, Cambridge university press, Cambridge, 1989.

17. N.H. Ibragimov, (ed.) CRC Hand book of Lie Group Analysis of Differential Equations, Vol. 2, CRC Press, Boca Rota, Florida, 1995.

18. N.H. Ibragimov, (ed.) CRC Hand book of Lie Group Analysis of Differential Equations, Vol. 3, CRC Press, Boca Rota, Florida, 1996.

19. L.V. Ovsianikov, Group Analysis of Differential Equations, Nauka, Moscow, 1978, (Engl. transl. Academic Press, New York, 1982.

20. Kamran Fakhar, Shagufta Rani, Zu-Chi Chen, Lie gruop analysis of axisymmetric flow, Chaos, Solitons and Fractals, 19, 1261 - 1267, 2004.

21. T. Hayat, A.H. Kara, E. Momoniat, Exact flow of a third-grade fluid on a porous wall, Int. J. Non-Linear Mechamics, 38, 1533 - 1537, 2003.

22. M. Yurusoy, Similarity solutions for creeping flow and heat transfer in second grade fluids, Int. J. Non-Linear Mechanics, 39, 665 - 672, 2004. 